\title{
FOSSIL REMNANTS OF REIONIZATION IN THE HALO OF THE MILKY WAY
}

\author{
P. Madau, ${ }^{1}$ M. Kuhlen, ${ }^{2}$ J. Diemand, ${ }^{1}$ B. Moore, ${ }^{3}$ M. Zemp,${ }^{1,4}$ D. Potter ${ }^{3}$ And J. Stadel ${ }^{3}$ \\ Received 2008 September 16; accepted 2008 October 21; published 2008 November 13
}

\begin{abstract}
Our recently completed one billion particle Via Lactea II simulation of a Milky Way-sized dark matter halo resolves over 50,000 gravitationally bound clumps orbiting today within the virialized region of the main host. About 2300 of these subhalos have one or more "progenitors" with $M>10^{6} M_{\odot}$ at redshift $z=11$, i.e., massive enough for their gas to have cooled via excitation of $\mathrm{H}_{2}$ and fragmented prior to the epoch of cosmic reionization. We count 4500 such progenitors: if these were able to convert a fraction of their gas content into very metalpoor stars with a Salpeter initial mass function (IMF), they would be shining today with a visual magnitude $M_{V}=6.7$ per solar mass in stars. Assuming a universal baryon fraction, we show that mean star formation efficiencies as low as $0.1 \%$ in progenitors $\ll 10^{8} M_{\odot}$ would overproduce the abundance of the faint Galactic dwarf spheroidals observed by the Sloan Digital Sky Survey. Star formation at first light must either have occurred with an IMF lacking stars below $0.9 M_{\odot}$, or was intrinsically very inefficient in small dark matter halos. If the latter, our results may be viewed as another hint that there is a minimum scale in galaxy formation.
\end{abstract}

Subject headings: cosmology: theory — dark matter — galaxies: dwarf — galaxies: formation — galaxies: halos

Online material: color figures

\section{INTRODUCTION}

Galaxy halos are one of the crucial testing grounds for structure formation scenarios, as they contain the imprints of past accretion events, from before the epoch of reionization to the present. In the standard $\Lambda \mathrm{CDM}$ concordance cosmology, objects like the halo of our Milky Way are assembled via the hierarchical merging and accretion of many smaller progenitors. Subunits collapse at high redshift, are dense, and have cuspy density profiles, and when they merge into larger hosts they are able to resist tidal disruption. Indeed, the Via Lactea Project, a suite of some of the largest cosmological simulations of Galactic dark matter substructure, has shown that galaxy halos today are teeming with surviving "subhalos" (Diemand et al. 2007, 2008).

The predicted subhalo counts vastly exceed the number of known satellites of the Milky Way, a "substructure problem" that has been the subject of many recent studies. While a full characterization of this discrepancy is hampered by luminosity bias in the observed satellite luminosity function (LF; Koposov et al. 2008), it is generally agreed that cosmic reionization may offer a plausible solution to the apparent conflict between the Galaxy's relatively smooth stellar halo and the extremely clumpy cold dark matter distribution. In this hypothesis, photoionization heating after reionization breakthrough reduces the star-forming ability of newly forming halos that are not sufficiently massive to accrete intergalactic gas (e.g., Bullock et al. 2000; Somerville 2002; Kravtsov et al. 2004; Ricotti \& Gnedin 2005; Moore et al. 2006; Strigari et al. 2007; Simon \& Geha 2007; Madau et al. 2008). The exact value of this mass threshold remains uncertain, as it depends on poorly known quantities such as the amplitude and spectrum of the ionizing background and the abil-

\footnotetext{
Department of Astronomy and Astrophysics, University of California, Santa Cruz, CA 95064; pmadau@ucolick.org, diemand@ucolick.org.

${ }^{2}$ Institute for Advanced Study, Einstein Drive, Princeton, NJ 08540; mqk@ias.edu.

${ }^{3}$ Institute for Theoretical Physics, University of Zurich, Winterthurerstrasse 190,8057Zurich, Switzerland; dpotter@physik.uzh.ch, moore@physik.uzh.ch, stadel@physik.uzh.ch.

${ }^{4}$ Astronomy Department, University of Michigan, Ann Arbor, MI 48109; mzemp@umich.edu.
}

ity of the system to self-shield against UV radiation (e.g., Efstathiou 1992; Dijkstra et al. 2004).

In this Letter we take a different approach and focus instead on the sources of reionization itself. We use our one billion particle Via Lactea II simulation to constrain the character of star formation (initial mass function and efficiency of gas conversion into stars) at first light, i.e., in subgalactic halos prior to the epoch of reionization. The starting point of our investigation is the finding that as many as 2300 bound clumps that survive today in the Via Lactea II halo have early progenitors that were massive enough for their gas to cool via excitation of molecular hydrogen, and to fragment before reionization breakthrough. If low-mass stars formed in the process, their hosts would be shining today as faint Milky Way satellites.

\section{VIA LACTEA II}

Our recently completed Via Lactea II simulation, one of the highest precision calculations of the assembly of the Galactic CDM halo to date (Diemand et al. 2008), offers the best opportunity for a systematic investigation of the fossil records of reionization in the halo of the Milky Way. Via Lactea II employs just over one billion $4100 M_{\odot}$ particles to model the formation of a $M_{200}=1.9 \times 10^{12} M_{\odot}$ Milky Way-sized halo and its substructure. It resolves 50,000 subhalos today within the host's $r_{200}=402 \mathrm{kpc}$ (the radius enclosing an average density 200 times the mean matter value). The Wilkinson Microwave Anisotropy Probe (WMAP) 5 year data require the universe to be fully reionized by redshift $z=11.0 \pm 1.4$ (Dunkley et al. 2008). Assuming that the region around the Milky Way was reionized at about the same epoch of the universe as a whole, we can trace the progenitors of present-day surviving substructure back to a time prior to reionization breakthrough, i.e., before star formation was quenched by photoheating. We use the 6DFOF group finder described in Diemand et al. (2007) to identify peaks in phase-space density at $z=11$ and 0 . The resulting groups contain between 16 and a few thousand particles linked together in the centers of halos and subhalos. Note that the gravitationally bound region often extends beyond this central group. We link a descendant $z=0$ group A to a high-redshift progenitor B if 

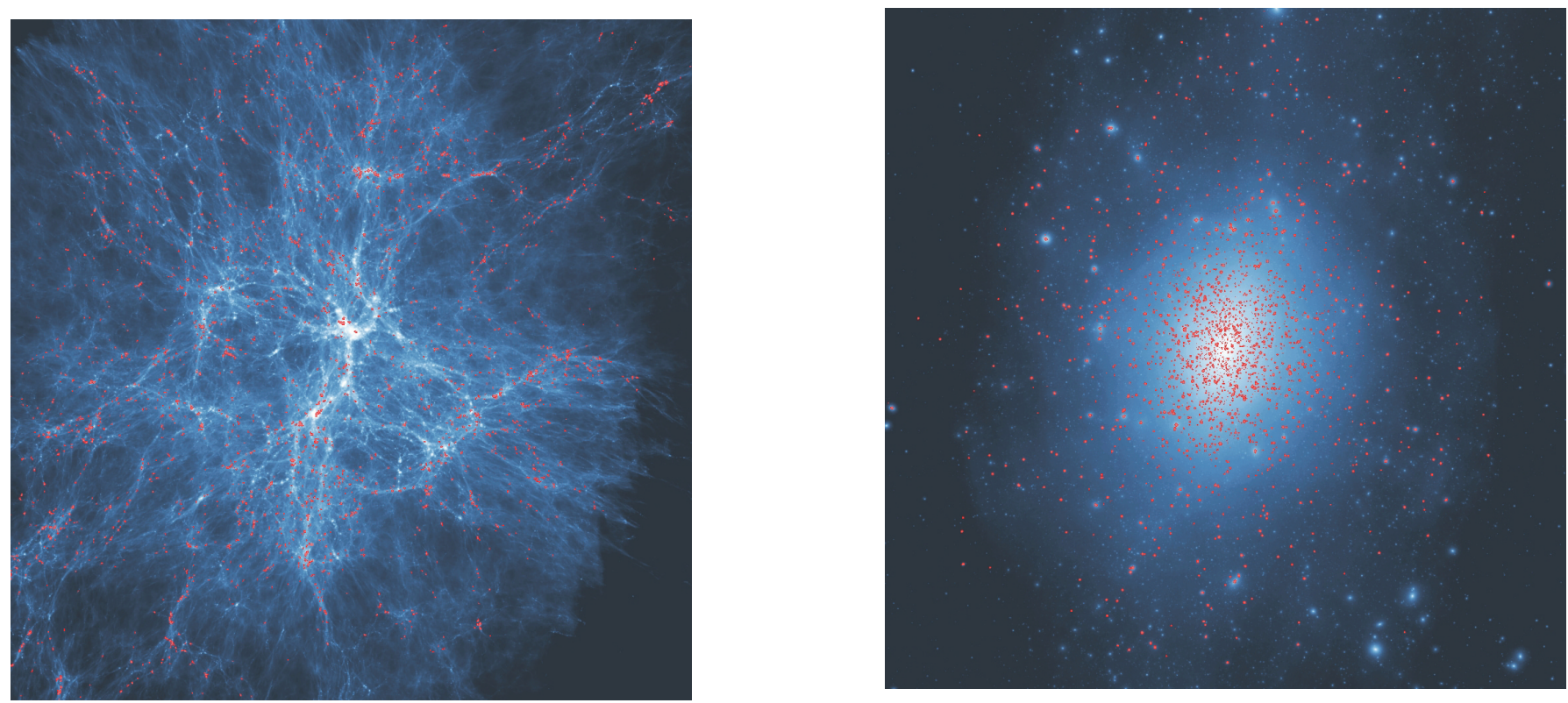

FIG. 1.-Left: Projected dark matter density map of Via Lactea II at $z=11$. The image covers 3.5 comoving Mpc across and shows (magenta) all progenitor minihalos with $M>10^{6} M_{\odot}$ that have a bound descendant today within $r_{200}$. Right: $z=0$ descendants of those early progenitors. The image covers 800 kpc across.

A contains more than 10 particles from $\mathrm{B}$ and more than any other descendant of B. Thus, a descendant can have more than one progenitor, but a progenitor is linked to at most one descendant. Using the halo centers provided by $6 \mathrm{DFOF}$ ensures that material from $\mathrm{B}$ does indeed contribute to the central regions of A, i.e., that a star cluster that formed in the core of a progenitor clump would end up today in the dwarf galaxy of a descendant subhalo.

We include in our analysis only progenitors that either survive individually during the hierarchical clustering process or con-

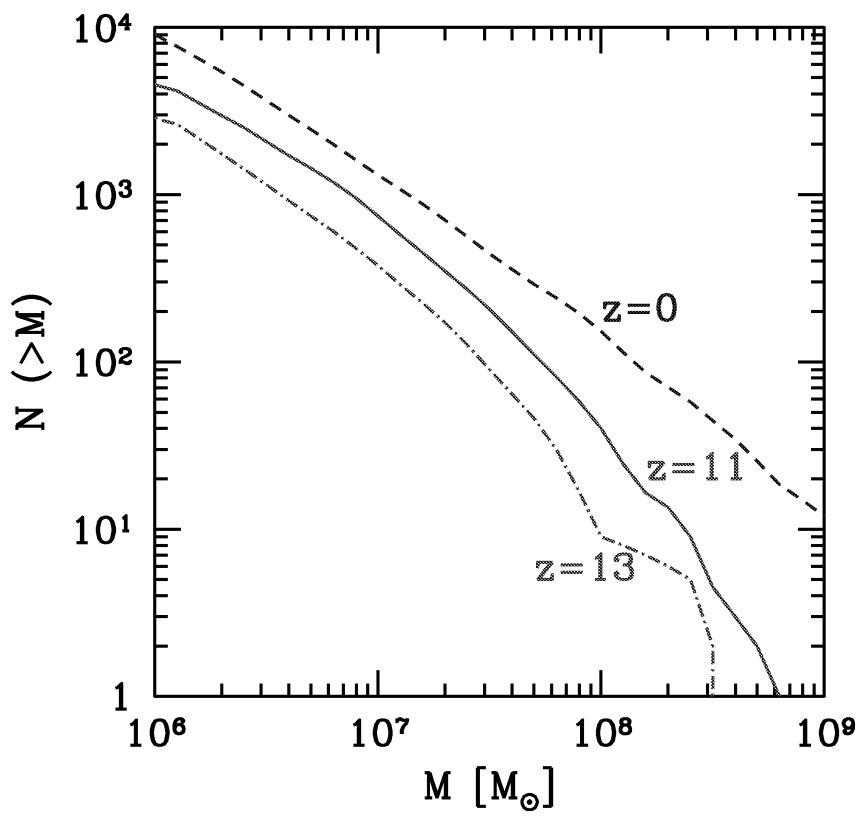

FIG. 2.-Via Lactea II cumulative substructure mass function at different epochs. Solid curve: All 4500 progenitor halos with $M>10^{6} M_{\odot}$ at $z=11$ that contribute to a gravitationally bound descendant (2300 of them) today within $r_{200}$. Dot-dashed curve: Same, for the $2900 M>10^{6} M_{\odot}$ progenitors at $z=13$. Also shown for comparison is the mass function of all Via Lactea II subhalos at the present epoch (dashed curve). [See the electronic edition of the Journal for a color version of this figure.] tribute to a gravitationally bound descendant at $z=0$. About 10,000 "first generation" systems above $10^{6} M_{\odot}$ are totally disrupted by tidal forces: for half of them, all of their particles lie today within $r_{200}$ and contribute to the smooth stellar halo. Figure 1 shows an image of all Via Lactea II progenitor halos above $10^{6} M_{\odot}$ at $z=11$ with a bound descendants within $r_{200}$ today, while Figure 2 shows the cumulative mass functions of progenitors and parents. We track forward in time 4500 of these early minihalos into 2300 descendants today. ${ }^{5}$

\section{STAR FORMATION AT FIRST LIGHT}

Cosmological hydrodynamics simulations of structure formation in a $\Lambda \mathrm{CDM}$ universe have found that in the early collapse of $\gtrsim 10^{6} M_{\odot}$ systems, enough $\mathrm{H}_{2}$ is produced to cool the gas and allow star formation within a Hubble time (e.g., Abel et al. 2002; Bromm et al. 2002). Figure 2 shows that a few hundred progenitors are above the "atomic cooling" mass threshold of $\sim 3 \times 10^{7} M_{\odot}\left(T_{\text {vir }} \gtrsim 10^{4} \mathrm{~K}\right)$, where gas can cool and fragment via excitation of hydrogen Ly $\alpha$. Numerical studies also suggest that, while primordial stars were likely very massive and formed in isolation at $z \gtrsim 20$ (e.g., O'Shea \& Norman 2007), low-mass second-generation stars can form as soon as a minimum preenrichment level of $Z=10^{-5 \pm 1} Z_{\odot}$ is reached (e.g., Schneider et al. 2002). The existence of $0.8 M_{\odot}$ extremely iron-deficient stars in the halo of the Milky Way (Christlieb et al. 2002; Frebel et al. 2005) indicates that low-mass star formation was possible at very low metallicities (e.g., Tumlinson 2007). In order to constrain the number of low-mass stars that lit up dark matter halos before reionization, our default model makes a number of simplifying assumptions. (1) All progenitor hosts above $M(z=11)=10^{6} M_{\odot}$ have a gas content given by the universal baryon fraction, $M_{\text {gas }}=\left(\Omega_{b} / \Omega_{m}\right) M=0.17 M$. (2) At $z \gtrsim 11$ a fraction $f_{*}$ of this gas is turned into very metal-poor stars with

${ }^{5}$ Note that the size of the Galaxy halo is not known very precisely, since the effects of disk formation on the total mass distribution are still poorly constrained. When comparing Via Lactea II to the Milky Way, this translates into an uncertainty in the abundance of subhalos of about a factor of 2 (Klypin et al. 2002; Dutton et al. 2007). 


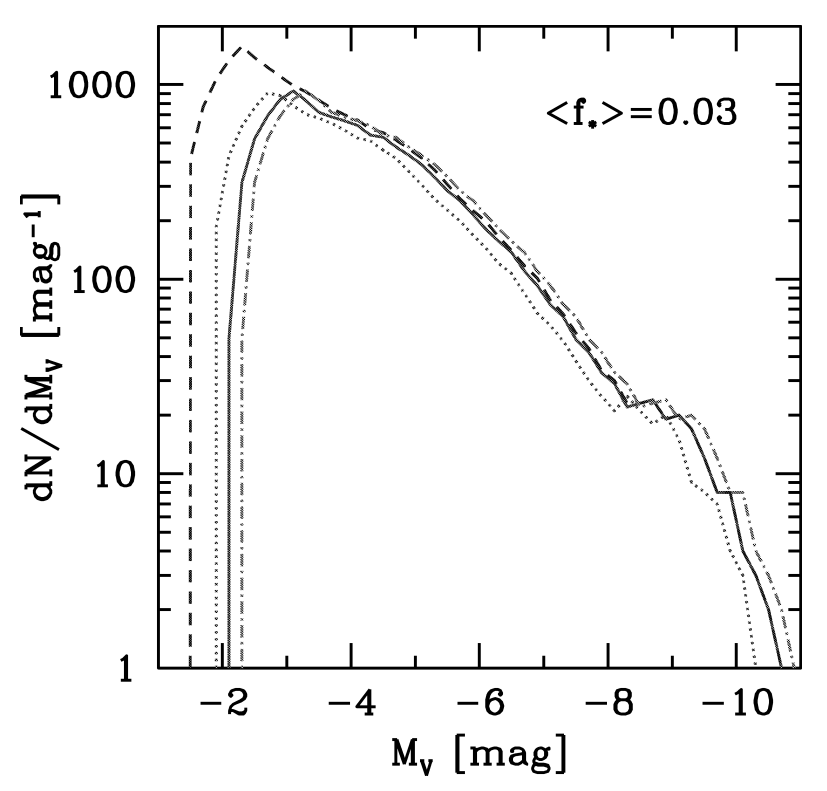

FIG. 3.-Luminous fossil remnants of reionization. Solid curve: Present-day LF of all Via Lactea II subhalos that had one or more progenitor with $M>$ $10^{6} M_{\odot}$ at $z=11$. At this epoch, a fraction $f_{*}$ of the gas content of each progenitor is turned instantaneously into stars with a Salpeter IMF and metallicity $Z=Z_{\odot} / 200$. Dashed curve: Same, for $M>5 \times 10^{5} M_{\odot}$. Dot-dashed curve: Same, for a Chabrier (2003) IMF. Dotted curve: Same, for $Z=Z_{\odot} / 5$. In all the above cases visual magnitudes at age $13.7 \mathrm{Gyr}$ have been calculated using Bruzual \& Charlot's (2003) models (1994 Padova tracks). [See the electronic edition of the Journal for a color version of this figure.]

$Z=Z_{\odot} / 200$ (the lowest metallicity allowed by Bruzual \& Charlot's [2003] stellar population synthesis models) and a standard Salpeter initial mass function (IMF) in the range $0.1<m_{*}<$ $100 M_{\odot}$. The star formation efficiency is independent of $M$. (3) Star formation is suppressed at later epochs in all progenitors and their descendants. (4) Primordial stellar systems are deeply embedded in progenitor minihalos and remain largely unaffected by tidal stripping even if their hosts are not. The complete tidal disruption of a host, however, also destroys its stellar system.

We are now in the position to construct the present-day LF of the fossil remnants of reionization in the halo of the Milky Way. According to Bruzual \& Charlot (2003), a stellar population undergoing an instantaneous burst will be shining at age 13.7 Gyr with a visual magnitude per solar mass in stars of $6.7 \mathrm{mag}$ (Salpeter IMF, $Z=Z_{\odot} / 200$ ). The steep LF of Via Lactea II firstgeneration halos predicted in this case is shown in Figure 3 for $\left\langle f_{*}\right\rangle=0.03$. Luminous remnants are distributed between $M_{V}=-2$ and -10 , and there are more than 200 relatively bright objects with $M_{V}<-6$, i.e., as bright as Boötes. A mean star formation efficiency 3 times smaller would shift this curve 1.2 mag to the left. The cutoff at faint magnitudes simply reflects our assumption that clumps below $M(z=11)=10^{6} M_{\odot}$ never form stars: the effect of lowering this mass threshold by a factor of 2 is also shown for comparison. Note that implausibly increasing the metallicity of early stars to $Z=Z_{\odot} / 5$ would only shift the curve $0.3 \mathrm{mag}$ to the left (Bruzual \& Charlot 2003). Similarly, a Chabrier (2003) IMF in the same mass range instead of Salpeter has only a small (0.2 mag) brightening effect.

\section{CONSTRAINTS FROM THE SDSS}

Over the last 2 years, the Sloan Digital Sky Survey (SDSS) has doubled the number of known Milky Way dwarf spheroidals (dSphs) brighter than $M_{V}=-2$ (e.g., Belokurov et al. 2007).

An accurate estimate of the total number of dwarfs requires a correction to the observed LF that accounts for completeness

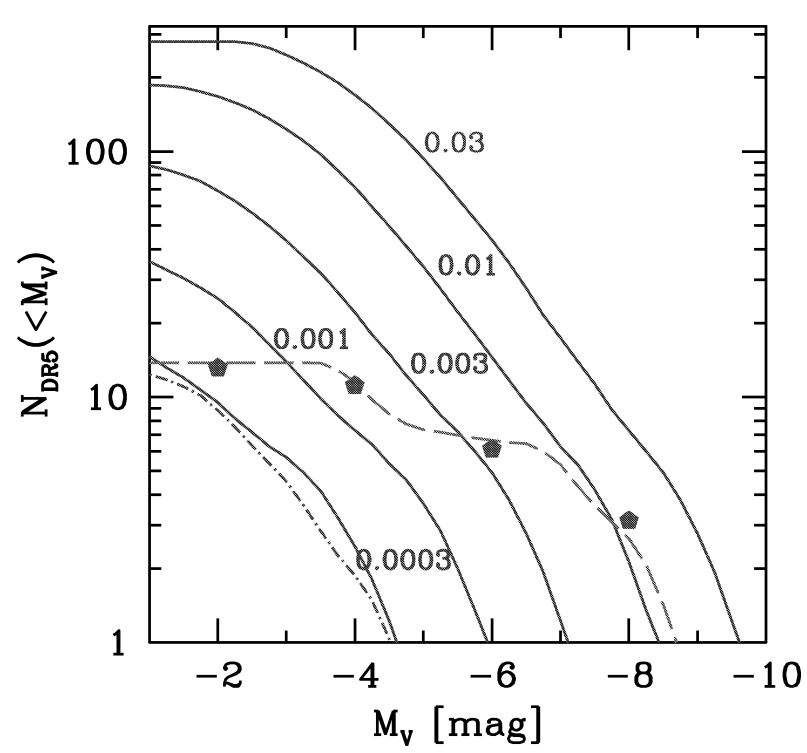

FIG. 4.-Via Lactea II first-generation $(z=11)$ subhalos detectable today by the SDSS DR5. All curves assume $M>10^{6} M_{\odot}$, a Salpeter IMF, and $Z=Z_{\odot} / 200$. The data points are taken from the compilation of Tollerud et al. (2008). Labeled solid curves: $\left\langle f_{*}\right\rangle=0.03,0.01,0.003,0.001$, and 0.0003 . Dashed curve: Mass-dependent star formation efficiency, $\left\langle f_{*}\right\rangle=(0.02$, $0.0025,0)$ for $M$ in the range $\left(>7 \times 10^{7}, 3.5 \times 10^{7}-7 \times 10^{7},<3.5 \times 10^{7} M_{\odot}\right)$. Also plotted for comparison is the LF of the remnants of $z=13$ halos with $\left\langle f_{*}\right\rangle=0.0005$ (bottom short dot-dashed curve). [See the electronic edition of the Journal for a color version of this figure.]

limits and that depends on the unknown intrinsic spatial distribution of sources. Rather than producing an "unbiased" list of Galactic satellites as in Tollerud et al. (2008), we apply here the completeness limit of the SDSS DR5 to our predicted fossil remnants in Via Lactea II (Fig. 3) to construct an artificial DR5 sample of luminous first-generation substructure. As computed by Koposov et al. (2008) and fitted by Tollerud et al. (2008), the maximum accessible distance beyond which an object of magnitude $M_{V}$ will go undetected by the SDSS is

$$
r_{\max }=\left(\frac{3}{4 \pi f_{\mathrm{DR} 5}}\right)^{1 / 3} \times 10^{\left(-0.6 M_{V}-5.23\right) / 3} \mathrm{Mpc},
$$

where $f_{\mathrm{DR} 5}=0.194$ is the fraction of the sky covered by DR5. Therefore, only subhalos brighter than $M_{V}=-6.6$ will be detected all the way to $r_{200}$ : first-generation remnants with $M_{V}=$ -4 will be included in the sample only if they are closer than $120 \mathrm{kpc}$. (The above detection limit applies to objects with surface brightness $\$ 30 \mathrm{mag} \operatorname{arcsec}^{-2}$, i.e., we implicitly assume that our luminous progenitors are less diffuse than this.) As a first-order correction, we also assume that subhalos within $r_{\max }$ are detected with $100 \%$ efficiency, and account for the partial sky coverage of SDSS by multiplying the number of detectable subhalos by $f_{\mathrm{DR} 5}$. The resulting cumulative $\mathrm{LF} N_{\mathrm{DR} 5}\left(<M_{V}\right)$ is plotted in Figure 4 for values of $\left\langle f_{*}\right\rangle$ that decrease from $3 \%$ down to $3 \times 10^{-4}$. In this range, the faint end of the predicted LF is dominated by the remnants of $10^{6}-10^{7} M_{\odot}$ progenitors. For an efficiency of $1 \%$, SDSS should have detected $\sim 150$ firstgeneration systems brighter than $M_{V}=-2$, while only a dozen are actually observed.

The known Milky Way satellites are also plotted for comparison; these include the SDSS 11 dwarfs with $M_{V}<-2$ (including Leo $\mathrm{T}$ which, at a distance of $417 \mathrm{kpc}$, lies just outside $r_{200}$ ) as well as the 11 (times $f_{\mathrm{DR} 5}$ to correct for the partial sky coverage) classical (pre-SDSS) bright satellites. Clearly, mean star formation efficiencies as low as $0.1 \%$ in progenitors below 
$10^{7} M_{\odot}$ would overproduce the abundance of ultrafaint $d S p h s$. The mean efficiency of star formation must actually scale with halo mass in order to reproduce the observations with first-generation remnants. This is because a constant efficiency translates into a steep LF that follows the steep mass function of CDM field halos. By contrast, the 23 known satellites of the Milky Way have a flat LF and shine with luminosities ranging from about $10^{3}$ to $10^{9}$ times solar. The dashed curve in Figure 4 shows a toy model with a mass-dependent efficiency, $\left\langle f_{*}\right\rangle=(0.02$, $0.0025,0)$ for $M$ in the range $M=\left(>7 \times 10^{7}, 3.5 \times\right.$ $10^{7}-7 \times 10^{7},<3.5 \times 10^{7} M_{\odot}$ ). Note how the data allow efficiencies of order $1 \%$ only above the atomic cooling threshold.

\section{SUMMARY}

In this work we have used results from the one billion particle Via Lactea II simulation to study for the first time the fossil signatures of the pre-reionization epoch in the Galactic halo and set constraints on the baryonic building blocks of today's galaxies. We have traced the progenitors of present-day surviving substructure back to a time prior to reionization breakthrough, i.e., before star formation was quenched by an external UV background. We have then populated early-forming minihalos with very metal-poor stars following a Salpeter IMF, and looked at the photometric properties today of their 2300 descendants within $r_{200}$. We have shown that even star formation efficiencies as low as $0.1 \%$ in progenitor minihalos below $10^{7} M_{\odot}$ would overproduce the abundance of Galactic dSphs observed by the SDSS. This value should be regarded as an upper limit, since luminous dSphs are known to have formed a fraction of their stars after reionization (e.g., Orban et al. 2008). It is also meant to be taken as a mean value, since an alternative possibility is to allow stars to form efficiently in a small fraction of minihalos at some given mass and to suppress star formation in the remainders, rather than reducing the efficiency across the board. Note that the parameter $f_{*}$ is degenerate with the baryon fraction: if the gas content of early virialized structure was smaller than the universal value (as found, e.g., by O'Shea \& Norman 2007), then our limits on the star formation efficiency would be correspondingly higher.
We can now test a posteriori the main assumption underlying our work, i.e., that early Via Lactea II progenitors were populated with "pre-reionization" stellar systems. In the simulated highresolution volume, there are $17,700 M>10^{6} M_{\odot}$ subhalos at $z=11$, spread over a comoving volume of $78 \mathrm{Mpc}^{3}$ containing a total mass of $M_{\mathrm{DM}}=3.5 \times 10^{12} M_{\odot}$. Stars distributed according to a Salpeter IMF produce during their lifetime $f_{\gamma} \approx$ 4000 Lyman continuum photons per stellar proton, of which only a fraction $f_{\text {esc }}$ will escape into the intergalactic medium (IGM). The total stellar mass in these early-forming progenitors is $M_{*}=3.6 \times 10^{10}\left\langle f_{*}\right\rangle M_{\odot}$. Hydrogen photoionization requires $\left(1+N_{\text {rec }}\right)$ photons above $13.6 \mathrm{eV}$ per atom, where $N_{\text {rec }}$ is the number of radiative recombinations over a Hubble time. The total mass of intergalactic gas that can be kept ionized is then $M_{\text {ion }}=f_{\gamma} M_{*} f_{\text {esc }} /\left(1+N_{\text {rec }}\right)$. The condition $M_{\text {ion }}<\left(\Omega_{b} / \Omega_{m}\right) M_{\mathrm{DM}}$ then implies $\left\langle f_{*}\right\rangle<0.004\left[\left(1+N_{\text {rec }}\right) / f_{\text {esc }}\right]$, where the factor in square brackets is of order 10 or so. The low star formation efficiencies derived in this work appear then to be consistent with the idea that the region around the Milky Way was reionized at $z \lesssim 11$ by external radiation, before stars in the Local Group formed in sufficient numbers (Weinmann et al. 2007). We conclude that star formation at first light either must have occurred with an IMF lacking stars below $0.9 M_{\odot}$, or was intrinsically very inefficient in small dark matter halos. If the former, this may be an indication of an upward shift in the mass scale of the IMF at early cosmological times owing, e.g., to the hotter cosmic microwave background (Larson 2005). If the latter, our results may be viewed as another hint (see Strigari et al. 2008) of a minimum scale in galaxy formation, below which supernova feedback (Dekel \& Silk 1986) and/or $\mathrm{H}_{2}$ photodissociation by a Lyman-Werner background (Haiman et al. 1997) sharply suppress star formation.

We thank J. Bullock and Z. Haiman for providing useful comments on an earlier draft. Support for this work was provided by NASA through grants HST-AR-11268.01-A1 and NNX08AV68G (P. M.) and Hubble Fellowship grant HST-HF01194.01 (J. D.). M. K. gratefully acknowledges support from the William L. Loughlin Fellowship at the Institute for Advanced Study.

\section{REFERENCES}

Abel, T., Bryan, G., \& Norman, M. L. 2002, Science, 295, 93

Belokurov, V., et al. 2007, ApJ, 654, 897

Christlieb, N., et al. 2002, Nature, 419, 904

Bromm, V., Coppi, P. S., \& Larson, R. B. 2002, ApJ, 564, 23

Bruzual, G., \& Charlot, S. 2003, MNRAS, 344, 1000

Bullock, J. S., Kravtsov, A. V., \& Weinberg, D. H. 2000, ApJ, 539, 517

Chabrier, G. 2003, ApJ, 586, L133

Dekel, A., \& Silk, J. 1986, ApJ, 303, 39

Diemand, J., Kuhlen, M., \& Madau, P. 2007, ApJ, 657, 262

Diemand, J., Kuhlen, M., Madau, P., Zemp, M., Moore, B., Potter, D., \& Stadel, J. 2008, Nature, 454, 735

Dijkstra. M., Haiman, Z., Rees, M. J., \& Weinberg, D. H. 2004, ApJ, 601, 666

Dunkley, J., et al. 2008, ApJS, submitted (arXiv:0803.0586)

Dutton, A. A., van den Bosch, F. C., Dekel, A., \& Courteau, S. 2007, ApJ, 654,27

Efstathiou, G. 1992, MNRAS, 256, 43P

Frebel, A., et al. 2005, Nature, 434, 871

Haiman, Z., Rees, M. J., \& Loeb, A. 1997, ApJ, 476, 458

Klypin, A., Zhao, H., \& Somerville, R. S. 2002, ApJ, 573, 597

Koposov, S., et al. 2008, ApJ, 686, 279
Kravtsov, A. V., Gnedin, O. Y., \& Klypin, A. A. 2004, ApJ, 609, 482

Larson, R. B. 2005, MNRAS, 359, 211

Madau, P., Diemand, J., \& Kuhlen, M. 2008, ApJ, 679, 1260

Moore, B., Diemand, J., Madau, P., Zemp, M., \& Stadel, J. 2006, MNRAS, 368,563

Orban, C., Gnedin, O. Y., Weisz, D. R., Skillman, E. D., Dolphin, A. E., \& Holtzman, J. A. 2008, ApJ, 686, 1030

O'Shea, B. W., \& Norman, M. L. 2007, ApJ, 654, 66

Ricotti, M., \& Gnedin, N. Y. 2005, ApJ, 629, 259

Schneider, R., Ferrara, A., Natarajan, P., \& Omukai, K. 2002, ApJ, 571, 30

Simon, J. D., \& Geha, M. 2007, ApJ, 670, 313

Somerville, R. S. 2002, ApJ, 572, L23

Strigari, L. E., Bullock, J. S., Kaplinghat, M., Diemand, J., Kuhlen, M., \& Madau, P. 2007, ApJ, 669, 676

Strigari, L. E., Bullock, J. S., Kaplinghat, M., Simon, J. D., Geha, M., Willman, B., \& Walker, M. G. 2008, Nature, 454, 1096

Tollerud, E. J., Bullock, J. S., Strigari, L. E., \& Willman, B. 2008, ApJ, 688, 277

Tumlinson, J. 2007, ApJ, 665, 1361

Weinmann, S. M., Macció, A. V., Iliev, I. T., Mellema, G., \& Moore, B. 2007, MNRAS, 381, 367 